\title{
Laser-induced fluorescence with tunable excimer lasers as a possible method for instantaneous temperature field measurements at high pressures: checks with an atmospheric flame
}

\author{
Peter Andresen, A. Bath, W. Gröger, H. W. Lülf, G. Meijer, and J. J. ter Meulen
}

\begin{abstract}
A new method for instantaneous temperature field measurements based on LIF studies of $\mathrm{OH}, \mathrm{O}_{2}$, and $\mathrm{H}_{2} \mathrm{O}$ in an open atmospheric flame with a tunable excimer laser is suggested. In this method the crucial problem of quenching at higher pressures is almost completely eliminated by excitation to a fast predissociating state. The various possible excitation and fluorescence processes that can be induced in the narrow tuning range of the $\mathrm{KrF}$ laser are characterized experimentally by excitation and dispersion spectra for the three molecules $\mathrm{OH}, \mathrm{O}_{2}$, and $\mathrm{H}_{2} \mathrm{O}$. Of particular importance is the large power of the $\mathrm{KrF}$ laser, which allows efficient excitation of even weak transitions. The fast predissociation of these molecules in connection with the powerful excitation laser suggests that instantaneous temperature field measurements should be possible at higher pressures.
\end{abstract}

\section{Introduction}

Temperature measurements with lasers are important in flames, flows, plasmas, discharges, and in motors, where thermometers cannot be used because interference with the probe has to be avoided. In recent years a number of different laser techniques have been used to measure temperatures. In the low pressure regime sensitive and powerful techniques, such as laser-induced fluorescence (LIF) ${ }^{1}$ or resonance-enhanced multiphoton ionization (REMPI), ${ }^{2}$ are well known and have been used extensively. However, these techniques have severe disadvantages at higher pressures, because the ions can no longer be extracted or because the fluorescence is quenched. Therefore other laser techniques, such as coherent anti-Stokes Raman scattering (CARS) ${ }^{3}$ or Rayleigh scattering $(\mathrm{RS})^{4}$ have been suggested and used instead. Whereas RS can be used only in rather special situations, ${ }^{4}$

G. Meijer and J. J. ter Meulen are with Catholic University, 6525 ED Nijmegen, The Netherlands; the other authors are with MaxPlanck-Institut for Stromungsforschung, D-3400 Gottingen, Feder-

al Republic of Germany.

Received 5 June 1987.

0003-6935/88/020365-14\$02.00/0.

(C) 1988 Optical Society of America.
$\overrightarrow{C A R S}$ has been used at higher pressures in different environments with considerable success. ${ }^{3}$ Although CARS seems to be the only realistic technique for temperature measurements at high pressures, it has an inherent disadvantage: temperatures can be determined only in a single point with one laser shot. In many applications temperature information is required instantaneously in a 2-D field or even in a 3-D volume, for example, in turbulent systems.

In LIF, fluorescence originates from all points in the probe that are illuminated by the laser. A strong laser allows the illumination of rather large 2-D arrays with sufficient intensity. Sufficient means that the fluorescence yield from a single point in the array is high enough to be measured. The emission from the different points in the array can be analyzed using photographic techniques as, for example, in planar laserinduced fluorescence (PLIF). ${ }^{5}$

The main drawback of LIF is quenching of fluorescence at higher pressures by numerous collisions, which occur within the radiative fluorescence lifetime $\tau_{r}$. Many attempts to correct for collisional quenching failed, because the effect depends sensitively on the laser-excited state and on the collision partners. ${ }^{6}$ In many technical environments (flames or motors) with a complicated gas composition and many different collision partners, a correction of LIF data becomes impossible.

In this paper a LIF method is used in which the effect of quenching is avoided simply by exciting the 
molecule to a fast predissociating state from which fluorescence is emitted only during the predissociation lifetime $\tau_{p}$. For sufficiently short predissociation lifetimes there will be no collisions and consequently no quenching within $\tau_{p}$, even at higher pressures. Because many molecules predissociate at shorter wavelengths, ${ }^{7}$ the method should be applicable to a relatively large number of molecules. For the case of $\mathrm{O}_{2}$, this idea has already been suggested. ${ }^{8}$

An important point of the present experiment is that it is done with a high power tunable $\mathrm{KrF}$ laser. This laser delivers $400 \mathrm{~mJ} /$ pulse in a bandwidth of $\approx 0.5$ $\mathrm{cm}^{-1}$. This is orders of magnitude more power than is available from other commercial lasers in this frequency range. With this laser a large sheet can be illuminated with sufficient intensity even for weak absorbers. This point has been discussed theoretically in a recent paper in which a broadband ArF laser is suggested to do LIF in the $\mathrm{O}_{2}$ Schumann-Runge bands. ${ }^{9}$

Although the laser is only tunable between 248.0 and $248.9 \mathrm{~nm}$, many different vibrational-rotational transitions of three important molecules $\left(\mathrm{OH}, \mathrm{H}_{2} \mathrm{O}\right.$, and $\mathrm{O}_{2}$ ) were found to lie in this narrow tuning range. For the first time LIF in the $\mathrm{OH}\left({ }^{2} \Sigma-{ }^{2} \Pi\right)$ absorption band has been done with the very weak $3-0$ vibrational transition. ${ }^{10}$ Here and in the remaining part of the paper vibrational transitions are denoted always by $v^{\prime}$ $v^{\prime \prime}$, i.e., in this case $v^{\prime}=3, v^{\prime \prime}=0$. A series of different rotational transitions were identified. Dispersion spectra from single laser prepared states show that rotational quenching within the predissociation lifetimes is small even at atmospheric pressures. In addition a number of different transitions in the Schumann-Runge bands of $\mathrm{O}_{2}\left(B^{3} \Sigma_{u}^{-}-X^{3} \Sigma_{g}^{-}\right)$have been excited. ${ }^{11}$ A series of rotational lines of different vibrational transitions $(0-6,1-6,2-7$, and a few more) have been identified and again dispersion spectra have been measured from single laser excited states. For $\mathrm{H}_{2} \mathrm{O}$ a series of rotational transitions is obtained by two-photon excitation to the $\tilde{C}^{1} B_{1}$ state, in which case the bound-free fluorescence from the $\tilde{C}^{1} B_{1}$ state to the repulsive $A^{1} B_{1}$ state ${ }^{12}$ is used to deduce populations. ${ }^{13,14}$ Despite the very large power $(>\mathrm{GW} /$ $\mathrm{cm}^{2}$ ) neither the $\mathrm{H}_{2} \mathrm{O}$ nor the $\mathrm{OH}$ transitions were saturated. In contrast, the $\mathrm{O}_{2}$ transitions were completely saturated at much lower power.

The excitation spectra have been modeled theoretically assuming a well-defined rotational temperature for the probe. According to these calcuations, we find that both $\mathrm{H}_{2} \mathrm{O}$ and $\mathrm{OH}$ are good candidates for measuring rotational temperatures. For $\mathrm{O}_{2}$ only a few lowlying rotational transitions can be excited with the $\mathrm{KrF}$ laser. Within a vibrational band the probed states are too close in energy to give good precision for temperature measurements. However, rotational temperatures can be determined with good precision if different vibrational bands are used. In addition $\mathrm{O}_{2}$ is nicely suited to determine vibrational temperatures. It is directly proved that the lifetime of the $\mathrm{OH}\left({ }^{2} \Sigma, v^{\prime}=\right.$ 3 ) state is so short that quenching is small in atmospheric conditions. The even shorter lifetimes for
$\mathrm{H}_{2} \mathrm{O}$ (Ref. 12) and $\mathrm{O}_{2}$ (Ref. 15) should allow temperature measurements up to $\approx 50$ bar.

It will be discussed that temperature field measurements are possible with one laser for the cases of $\mathrm{OH}$ (rotational temperatures) and $\mathrm{O}_{2}$ (vibrational and rotational temperatures) by spectrally dispersing the fluorescence. $\mathrm{H}_{2} \mathrm{O}$ requires, similar to other methods, two delayed lasers and fast detection schemes.

It should also be mentioned that fluorescence trapping is no problem in the above cases. The transitions to the $v^{\prime}=3$ state in $\mathrm{OH}$ are only strong if they start from excited vibrational states. Because these excited vibrational states are hardly populated, trapping will be negligible. Similarly, in $\mathrm{O}_{2}$ only a few highly vibrationally excited molecules can absorb the fluoresence radiation. In the case of $\mathrm{H}_{2} \mathrm{O}$ the fluorescence is bound free and therefore reabsorption is impossible.

The following results are selected to demonstrate that the suggested method works from a spectroscopic point of view. Quantitative temperature measurements are not the main aim of this paper.

\section{Method}

In a gas with thermal equilibrium among rotational states, the relative density $n_{i}$ in a quantum state $i$ of a molecule $A B$ depends on the temperature $T$ via the Boltzman distribution:

$$
n_{i} \alpha(2 j+1) \exp \left(-E_{i} / k T\right),
$$

with $k$ is the Boltzmann constant, $E_{i}$ is the energy of quantum state $i$ above the ground state, and $j$ is the total angular momentum.

In LIF a molecule $A B$ is excited with a laser at some frequency $\nu_{1}$ from an initial state $i_{1}$ in the electronic ground state to a final state $f_{1}$ in some electronically excited state. The subsequent fluorescence intensity from $f_{1}$ is measured, for example, with a phototube. If collisions can be neglected, the fluorescence signal $S_{1}$ is proportional to the density $n_{1}$ of $A B$ molecules in quantum state $i_{1}$. The proportionality factor $P$ contains the line strength for absorption and emission and can also depend on directional properties of absorbed and emitted light. If the excitation laser is tuned to another frequency $\nu_{2}$, the density $n_{2}$ in another quantum state $i_{2}$ can be determined via the fluorescence signal $S_{2}$.

Because the fluorescence signals are proportional to the densities, the measured ratio $R=S_{1} / S_{2}$ is related to the temperature $T$ by

$$
R=\frac{S_{1}}{S_{2}}=\frac{P_{1} n_{1}}{P_{2} n_{2}}=\frac{P_{1}}{P_{2}} \frac{\left(2 j_{1}+1\right)}{\left(2 j_{2}+1\right)} \exp \left(\frac{E_{2}-E_{1}}{k T}\right),
$$

which can be inverted to give the temperature in terms of the measured ratio $R$ and the known quantities $E_{i}, j_{i}$, and $P_{i}$ :

$$
T=\frac{E_{2}-E_{1}}{k \ln \left[\frac{P_{2}\left(2 j_{2}+1\right)}{P_{1}\left(2 j_{1}+1\right)} R\right]} .
$$

An important advantage of such temperature measurements is that only relative fluorescence signals are 
required. Complicated calibration procedures are not necessary and laser power fluctuations are eliminated in a natural way.

To evaluate Eq. (2) the fluorescence signals $S_{1}$ and $S_{2}$ have to be measured separately. This can be achieved either by spectral filtering or by time resolution.

Spectral filtering is possible if the fluorescence from the electronically excited states $f_{1}$ and $f_{2}$ is sufficiently separated in wavelength. Then filters, prisms, or spectrometers can be used to measure $S_{1}$ and $S_{2}$ separately. In the present case this is possible for rotational and vibrational temperatures of $\mathrm{O}_{2}$ and rotational temperatures of $\mathrm{OH}$. The important advantage of spectral filtering is that only one laser may be required for temperature measurements, for example, if the laser excites both transitions simultaneously.

If the fluorescence from the states $f_{1}$ and $f_{2}$ is not sufficiently separated in wavelength, separation can be achieved via time resolution. In this case two lasers are fired with a small time delay and the fluorescence is recorded separately in time. This method has to be used for $\mathrm{H}_{2} \mathrm{O}$, because the emission is spectrally broad and independent of the rotational state.

In principle, the temperature can be determined from the density in any two different quantum states. The precision of the temperature measurement will however depend on the change of the measured quantity $R$ with temperature. For example, a small energetic difference between the two states will result in poor precision.

As discussed above, temperature measurements by LIF are based on the linearity of the fluorescence signal with the density in the probed state. In the low pressure regime (typically below $100 \mu$ bar) there are no collisions within the radiative lifetime $\tau_{r}$ and the linearity holds strictly. In this range the LIF technique is well established. Numerous experiments have been done, for example, to analyze rotational state distributions from chemical reactions, inelastic collisions, or photodissociation experiments.

At higher pressures the laser excited molecules will however collide with the ambient gases within $\tau_{r}$. The importance of secondary collisions may be illustrated by the example of an open atmospheric flame. A laser excited molecule with a typical fluorescence lifetime of $100 \mathrm{~ns}$ will undergo $\sim 1000$ hard sphere collisions during its radiative lifetime. The collisions can lead to energy transfer within the excited state or to fluorescence quenching.

Rotational and vibrational energy transfer within the excited state alone do not cause the most severe problems, because a photon is still emitted after excitation and the linearity between fluorescence signal and density is the same. Fluorescence quenching leads, however, to a deexcitation of the electronically excited state, such that no fluorescence photon is emitted. Although the fluorescence signal will still depend linearly on density (more particles yield more fluorescence), the linearity between fluorescence signal and density can be different for two different states $i_{1}$ and $i_{2}$. This is the case if fluorescence quenching is different for the corresponding excited states $f_{1}$ and $f_{2}$. In a number of studies it has been shown that quenching depends indeed sensitively both on the rotational level of the excited electronic state and on the collision partners. ${ }^{6}$ Fluorescence quenching is the main problem in the application of LIF at higher pressures, in particular for complicated gas compositions.

Several methods have been suggested to eliminate these problems. For example, the same upper state may be used in the excitation, i.e., $f_{1}=f_{2} .{ }^{16}$ Then quenching will still occur, but it will give the same reduction in fluorescence for the quantum states $i_{1}$ and $i_{2}$. The ratio of fluorescence signals will be related to the temperature in the same way as in Eq. (2). Howev$\mathrm{er}$, the only way to measure the fluorescence signals $S_{1}$ and $S_{2}$ separately is by time resolution, i.e., the method requires two tunable lasers. In addition the same upper state can only be reached from neighboring lower states, i.e., states with $\Delta j=2$. The method yields inherently low precision, because of the small energetic difference between the probed states.

In the present LIF method a molecule is excited with a strong laser to a fast predissociating state with a short lifetime $\tau_{p}$. Because the excited state molecules radiate only in the short time $\tau_{p}$, only a very small part of the molecules does fluoresce. This causes a tremendous loss in LIF signal, which is partly compensated, because the densities are much higher than in typical LIF experiments. The main advantage of this method is, however, that there will be no collisions - and consequently no quenching-within the predissociation lifetime, if $\tau_{p}$ is short enough. For example, the probability for a hard sphere collision within $\tau_{p}$ is $\sim 5 \times 10^{-2}$ in atmospheric conditions, if we assume a predissociation lifetime of 5 ps. For sufficiently short predissociation lifetimes quenching is impossible. However, in many cases predissociation is known to be quantum state dependent. A calibration procedure can be used to overcome this problem.

In this paper we demonstrate that the idea of the method works in atmospheric flames for $\mathrm{OH}, \mathrm{O}_{2}$, and $\mathrm{H}_{2} \mathrm{O}$ using the tunable $\mathrm{KrF}$ laser. $\mathrm{OH}, \mathrm{H}_{2} \mathrm{O}$, and $\mathrm{O}_{2}$ will not be the only molecules which can be used. In most cases diatomic molecules predissociate faster for shorter excitation wavelengths. With a strong lasers (for example, other tunable excimers and intense Stokes and anti-Stokes lines that can be generated from them) even weak transitions can be excited efficiently enough to obtain reasonable fluorescence intensity.

\section{Experimental Details}

The experimental setup is shown in Fig. 1. The basic technique is spectrally dispersed LIF. A KrF excimer laser (Lambda Physik EMG 150 EST) with a grating tuned oscillator is used for excitation. ${ }^{13}$ Several different lenses are used to focus the laser, but all results in this paper are for a $100-\mathrm{cm}$ focal length. Most measurements are made a few centimeters (3-5) away from the focus to avoid problems with undefined 


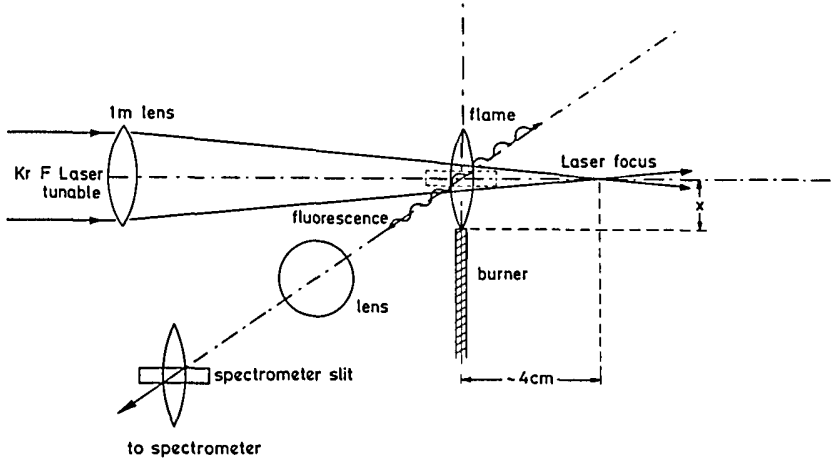

Figure 1. Experimental setup. The $\mathrm{KrF}$ laser is focused with a 100 -cm lens. The flame is installed $\sim 4 \mathrm{~cm}$ before the focus. The flame is adjusted relative to the laser. The fluorescence volume is given by the section of the flame that is imaged to the spectrometer slit by the quartz lens.

breakdowns in air. No reasonable results could be obtained in the focus itself.

We studied several different atmospheric flames $\left(\mathrm{O}_{2}-\mathrm{H}_{2}\right.$, butane, and acetylene $)$ generated by burners that are used for welding purposes otherwise. The flames are inserted in the excimer laser beam path at different distances between laser beam and burner tip (see Fig. 1). The main origin of noise in the spectra is from instabilities of the flame and pulse-to-pulse inistabilities in the laser power.

The fluorescence is sampled with a lens from a thin $\approx 10$-mm long line through the flame. Because a 1:1 imaging optics is used the fluorescence volume is essentially the image of the $10-\mathrm{mm}$ long entrance slit of the spectrometer (see Fig. 1). This setup is certainly not adequate for quantitative temperature measurements. Large differences in local temperatures may be expected along the fluorescence line, in particular for the flames used here. Because fluorescence is sampled from spots with different temperatures, the net result is an averaging over different temperatures along the fluorescence line through the flame. This implies that the resulting rotational distributions do not even have to be Boltzmann distributions. ${ }^{17}$

The fluorescence light is spectrally dispersed by a 0.3-m spectrometer (McPherson 218). The spectral dispersion is very important, because it allows discrimination among the fluorescence of $\mathrm{OH}, \mathrm{O}_{2}$, and $\mathrm{H}_{2} \mathrm{O}$. In some cases rather narrow slits are necessary for sufficient discrimination. The intensity of the dispersed light is measured with a cooled photomultiplier (RCA 31034) averaged with a boxcar and recorded on a strip chart recorder. A narrow boxcar gate is used to suppress direct emission from the flame. Because most of the fluorescence is emitted during the laser pulse, within the predissociation lifetime, this causes no loss in the LIF signal. Saturation of the PMT, due to the permanent background of the flame, was in some cases eliminated by a gating technique. ${ }^{18}$

Two basically different types of experiment are done. In the first type, the tunable $\mathrm{KrF}$ laser is fixed in frequency, inducing a particular transition of either $\mathrm{H}_{2} \mathrm{O}, \mathrm{O}_{2}$, or $\mathrm{OH}$. Then the spectrometer is scanned and the fluorescence intensity is recorded. In this way dispersion spectra from single rovibrational states are obtained. In the second type, the spectrometer is fixed to transmit radiation in a wavelength range where only one of the molecules fluoresces. Then the $\mathrm{KrF}$ excitation laser is scanned and the fluorescence intensity is recorded again. If the spectrometer resolution is good enough, the fluorescence of only one molecule is transmitted and excitation spectra of the corresponding molecule are obtained separately.

First, a simple Bunsen burner is installed in the beam path and the continuous emission of the flame is measured without laser and without boxcar. The dominant emission is due to $\mathrm{OH}\left({ }^{2} \Sigma, v^{\prime}=0,1\right)$. With a narrow boxcar gate this continuous emission is almost completely suppressed, i.e., with the use of a boxcar there is negligible background from the flame. If this flame is irradiated with the unlocked, i.e., broadband $\mathrm{KrF}$ laser, an intense fluorescence appears in the wavelength range from 250 to $550 \mathrm{~nm}$. This fluorescence is laser induced and originates from simultaneous exctitation of many different transitions in different molecules. It should be kept in mind that, although the laser is operated broad band, the spectral brightness $\left(\approx 3 \mathrm{~mJ} / \mathrm{cm}^{-1}\right)$ is still high compared to typical commercial tunable laser systems in this frequency regime. Therefore many molecular transitions can be excited simultaneously with reasonable efficiency. Although many different species might be excited in a flame with this strong laser, most of the fluorescence intensity turns out to be due to a number of different transitions in $\mathrm{OH}, \mathrm{O}_{2}$, and $\mathrm{H}_{2} \mathrm{O}$. Similar experiments have been done with a broadband $\mathrm{ArF}$ laser on $\mathrm{NO}$ and $\mathrm{O}_{2} \cdot{ }^{19}$

Considerable care is necessary to record excitation and dispersion spectra separately for different molecules. The selection of the appropriate wavelength range with the spectrometer is very important for the separation of excitation spectra. Because fluorescence of different molecules may be transmitted in the same wavelength range, the excitation spectra can contain lines of more than one molecule. Both the central wavelength and the resolution of the spectrometer are crucial for discrimination between the fluorescence of different molecules. Low resolution leads to insufficient separation, whereas for high resolution the excitation spectra may consist of single rotational lines (as will be seen later in Fig. 5). Only with moderate resolution, high enough to discriminate between the fluorescence of different molecules, but low enough to transmit, for example, all rotational lines of a vibrational transition, separate excitation spectra are obtained for $\mathrm{OH}, \mathrm{O}_{2}$, and $\mathrm{H}_{2} \mathrm{O}$. Nevertheless, a few small lines in the following spectra are due to insufficient spectral resolution.

The relative intensities of different rotational lines in the excitation spectra have to be considered with care. The fluorescence intensity from different excited states may be reduced if the transmission of the spectrometer is optimized for one specific line, i.e., the 
Table I. Absorption Lines for the Vibrational Transition 3-0 in the $A^{2} \sum-$ $X^{2} I I$ Absorption Band of $\mathrm{OH}$ that are in the Tuning Range of the KrF Laser (from Ref. 10) ${ }^{a}$

\begin{tabular}{lcc}
\hline $\begin{array}{l}\text { Rotational } \\
\text { transition }\end{array}$ & $\begin{array}{c}\text { Excitation } \\
\text { frequency } \\
\left(\mathrm{cm}^{-1}\right)\end{array}$ & $\begin{array}{c}\text { Transition } \\
\text { probability }\end{array}$ \\
\hline$P_{1}(9)$ & 40193.35 & 5.11 \\
$R_{2}(15)$ & 40212.91 & 7.15 \\
$Q_{2}(11)$ & 40229.17 & 10.53 \\
$Q_{12}(11)$ & 40231.40 & 0.32 \\
$R_{21}(15)$ & 40242.39 & 0.33 \\
$R_{1}(15)$ & 402.45 .39 & 7.52 \\
$P_{2}(8)$ & 40248.48 & 3.81 \\
$P_{12}(8)$ & 40249.94 & 0.58 \\
$Q_{21}(11)$ & 40263.17 & 0.33 \\
$Q_{1}(11)$ & 40265.40 & 11.55 \\
$Q_{12}(6)$ & 40278.82 & 0.21 \\
$P_{1}(8)$ & 40296.25 & 4.60 \\
$R_{2}(14)$ & 40314.98 & 6.65 \\
$Q_{2}(10)$ & 40319.47 & 9.50 \\
$Q_{12}(10)$ & 40321.53 & 0.35 \\
\hline
\end{tabular}

${ }^{a}$ The transition probabilities are $\left(2 J^{\prime \prime}+1\right)$ times the appropriate Honl-London factor. ${ }^{10}$

Table II. Absorption Lines of the $\mathrm{O}_{2}$ Schumann-Runge Bands $B^{3} \sum_{u^{-}}^{-}$ $x^{3} \sum^{-}$that are in the Tuning Range of the KrF Laser ${ }^{\mathrm{a}}$

\begin{tabular}{llll}
\multicolumn{4}{c}{$X^{3} \sum_{g}$ that are in the Tuning Range of the KrF Laser } \\
\hline $0 \leftarrow 4$ & & $1 \leftarrow 6$ & \\
$R(75)$ & 40199.0 & $P(37)$ & 40235.1 \\
$P(71)$ & 40302.4 & $R(39)$ & 40272.4 \\
& & $P(35)$ & 40317.4 \\
$0 \leftarrow 5$ & & $2 \leftarrow 6$ & \\
$R(55)$ & 40218.4 & $R(53)$ & 40206.8 \\
$P(51)$ & 40282.8 & $P(49)$ & 40280.5 \\
$R(53)$ & 40334.0 & $R(51)$ & 40321.2 \\
$0 \leftarrow 6$ & & $2 \leftarrow 7$ & \\
$R(19)$ & 40199.9 & $R(15)$ & 40199.0 \\
$P(15)$ & 40217.4 & $P(11)$ & 40215.2 \\
$R(17)$ & 40235.9 & $R(13)$ & 40227.3 \\
$P(13)$ & 40251.4 & $P(9)$ & 40241.1 \\
$R(15)$ & 40267.7 & $R(11)$ & 40251.3 \\
$P(11)$ & 40281.2 & $P(7)$ & 40262.7 \\
$R(13)$ & 40295.3 & $R(9)$ & 40270.9 \\
$P(9)$ & 40306.7 & $P(5)$ & 40279.9 \\
$R(11)$ & 40318.5 & $R(7)$ & 40286.2 \\
$P(7)$ & 40328.0 & $P(3)$ & 40292.8 \\
$R(9)$ & 40337.6 & $R(5)$ & 40297.2 \\
& & $P(1)$ & 40301.4 \\
& & $R(3)$ & 40303.8 \\
& & $R(1)$ & 40306.1 \\
\hline
\end{tabular}

${ }^{a}$ For different vibrational transitions $v^{\prime} \leftarrow v^{\prime \prime}$ the excitation frequency for the corresponding rotational transitions is given in $\mathrm{cm}^{-1}$.

fluorescence intensity partly reflects the fixed spectrometer bandpass.

In all the excitation spectra, the base line intensity increases at the left and right side of the tuning range. This experimental artifact originates from decreasing locking efficiency of the $\mathrm{KrF}$ laser at the end of the tuning range. This is discussed in detail in Ref. 13.

To obtain separate dispersion spectra, the linewidth for absorption is important. The absorption linewidth is determined by the linewidth of the laser or by the linewidth of the molecular transition. Many different transitions can be excited in the tuning range
Table III. For the $0 \leftarrow 6$ and $2 \leftarrow 7$ Excitation Processes in the $\mathrm{O}_{2}$ Schumann-Runge Bands, the Wavelength Range for Emission Subsequent to Excitation of One of the lines from Table II to Various Lower Vibrational States $v^{\prime \prime}$ is Given Together with the Relative Fluorescence Intensity ${ }^{a}$

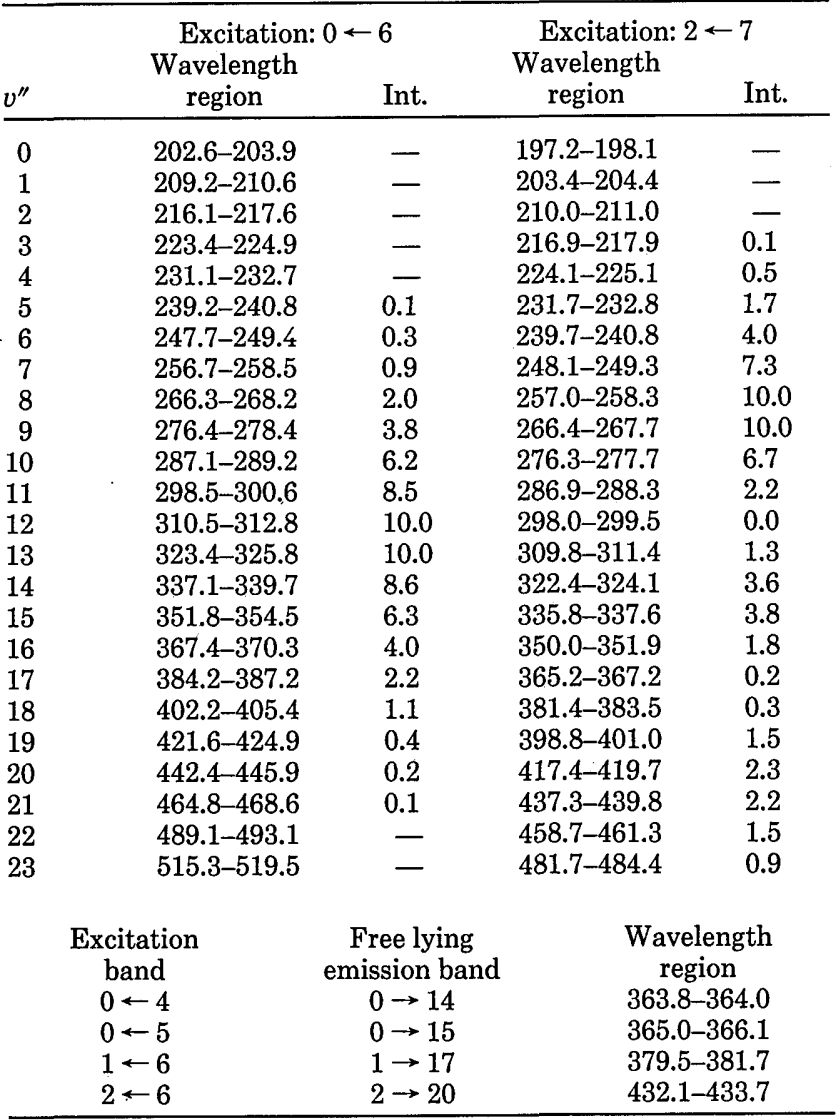

$a$ The indicated wavelength region covers all fluorescence from the different rotational lines that can be excited with the $\mathrm{KrF}$ laser. Because only relatively low rotational states are probed, the subsequent emission is restricted to a narrow range. The fluorescence intensity is calculated by $\nu^{3} \cdot q_{v^{\prime} v^{\prime \prime}}$ with the emission frequency $\nu$ and the FC factors from Ref. 20. The strongest emission is arbitrarily set to 10. For the other vibrational transition, only the best wavelength range for separate detection is given.

of the $\mathrm{KrF}$ laser and many of the lines are considerably broad, so that almost every frequency of the $\mathrm{KrF}$ laser contributes to some excitation process. This is why in some cases different transitions are excited simultaneously and why the dispersion spectra may contain contributions from different excited states of different molecules.

Systematic calculations have been performed to determine the transitions of $\mathrm{O}_{2}, \mathrm{OH}$, and $\mathrm{H}_{2} \mathrm{O}$ that are within the tuning range of the $\mathrm{KrF}$ laser (248.0-248.9 $\mathrm{nm})$. The most important absorption lines are given in Table $\mathrm{I}$ for $\mathrm{OH}$ and in Table II for $\mathrm{O}_{2}$. For $\mathrm{H}_{2} \mathrm{O}$ the lines are given in Ref. 13. For the excitation lines of $\mathrm{O}_{2}$ the wavelengths of all possible subsequent emission lines have been determined and the corresponding wavelength ranges are given in Table III for different lower vibrational states $v^{\prime \prime}$. The calculated spectra prove the unambiguous assignment of the following excitation and dispersion spectra. 
The calculated $\mathrm{O}_{2}$ dispersion spectra are described briefly. The spectra originate essentially from single laser prepared states in $B^{3} \Sigma_{u}^{-}$. No effects of secondary collisions are seen, because the lifetime in $B^{3} \Sigma_{u}^{-}$is so short. This is why the spectra can be fitted using only spectroscopic data. The measured (dispersed) fluorescence signal from a single prepared state $v^{\prime}, N^{\prime}$ in the excited eletronic state $B^{2} \Sigma_{u}^{-}$to various lower states $v^{\prime \prime}$, $N^{\prime \prime}$ in the electronic ground state $X^{3} \Sigma_{g}^{-}$is given by

$$
S=N_{0} \nu^{3} q_{v^{\prime} v^{\prime \prime}} \cdot H_{L} \cdot \tau_{p}^{v^{\prime \prime}} \cdot f(\lambda),
$$

with $N_{0}$ the number of molecules prepared, the emission frequency $\nu$ for the transition $v^{\prime}, N^{\prime} \rightarrow v^{\prime \prime}, N^{\prime \prime}$, the Franck-Condon factors $q_{v^{\prime} v^{\prime \prime}}$, the Hönl-London factor $H_{L}$, the (vibrationally state-dependent) predissociation lifetime $\tau_{p}^{v^{\prime \prime}}$, and the quantum efficiency $f(\lambda)$ of the detection system. The $\mathrm{O}_{2} / \mathrm{FC}$ factors are from Ref. 20 , the Hönl-London factors are $\left(N^{\prime}+1\right) /\left(2 N^{\prime}+1\right)$ for $P$ and $\left(N^{\prime}\right) /\left(2 N^{\prime}+1\right)$ for $R$ lines in the case of unresolved fine structure (see Ref. 21), the predissociation lifetimes are from Ref. 9, and the quantum efficiency of the detection system has been calibrated recently. ${ }^{14}$

\section{Results}

In the following, the excitation and dispersion spectra are discussed in the sequence $\mathrm{OH}, \mathrm{O}_{2}$, and $\mathrm{H}_{2} \mathrm{O}$ in qualitative agreement with the relative strength of emission. In most cases $\mathrm{OH}$ was the strongest emitter followed by $\approx 10$ times less intense contributions from the $0-6$ transition in $\mathrm{O}_{2}$ and another factor of 10 less $\mathrm{O}_{2}$ from the $2-7$ or $1-6$ transition. For $\mathrm{H}_{2} \mathrm{O}$ the fluorescence was even weaker, in particular because it is extended over a wider wavelength range. ${ }^{13,14}$ Then some temperature measurements are done in different positions in the flame for $\mathrm{OH}$ and $\mathrm{O}_{2}$, using the method described above. Finally it is shown that some qualitative insight can also be obtained for $\mathrm{OH}$ vibrational energy transfer within the excited state.

\section{A. OH Spectra}

Figure 2 shows an $\mathrm{OH}$ excitation spectrum with the spectrometer set to $329 \mathrm{~nm}$ and a resolution of $\approx 1 \mathrm{~nm}$. The spectrum is for an atmospheric $\mathrm{O}_{2}-\mathrm{H}_{2}$ flame and is recorded $\approx 10 \mathrm{~mm}$ above the burner tip and the boxcar averaged over three laser pulses. In the selected wavelength range mainly the $\mathrm{OH}\left({ }^{2} \Sigma^{+}, v^{\prime}=3 \rightarrow{ }^{2} \Pi, v^{\prime \prime}=3\right)$ $Q$-branch emission is monitored. All lines in Fig. 2 can be identified on the basis of the known $\mathrm{OH}$ spectrosco$\mathrm{py}^{10}$ and the corresponding identification of rotational transitions is given in the figure. The spectral resolution is obviously sufficient to discriminate against the $\mathrm{O}_{2}$ fluorescence.

The spectrum is almost noise free with a signal-tonoise ratio of $\approx 10^{4}$. Because the fluorescence volume is only $\approx 10^{-2} \mathrm{~cm}^{3}$, enough fluorescence will be already obtained from a much smaller fluorescence volume. This is of importance if larger sheets are used in temperature field measurements.

Figure 2 represents the first LIF spectrum in which the 3-0 transition has been excited with a laser. This very weak transition has not been excited before, be-

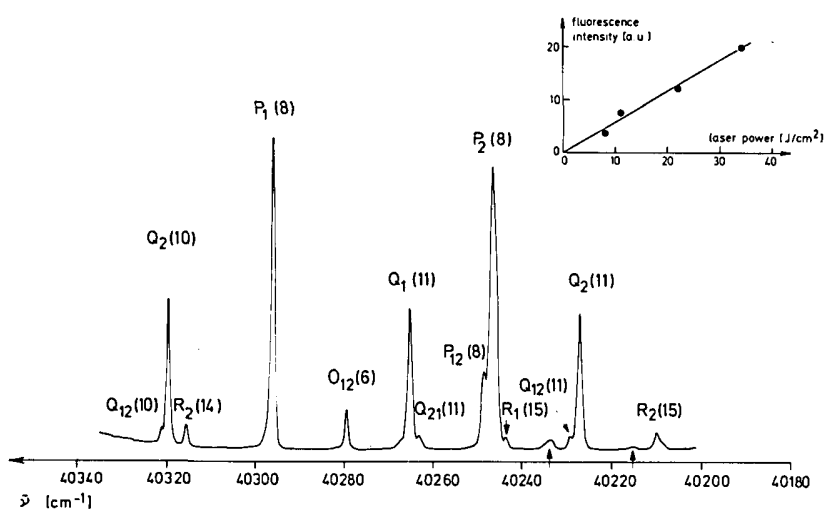

Figure 2. $\mathrm{OH}$ excitation spectrum $A^{2} \sum^{+}, v^{\prime}=3 \leftarrow X^{2} \Pi, v^{\prime \prime}=0$. The excitation of $\mathrm{OH}$ proceeds via the ${ }^{2} \sum-2 \Pi$ absorption band. The $3-0$ vibrational transition is excited; the excitation frequencies are given in $\mathrm{cm}^{-1}$. The spectrometer is set to $329 \mathrm{~nm}$ with a resolution of $\approx 1 \mathrm{~nm}$. The inset shows the linearity of fluorescence with laser power for the $P_{1}(8)$ line. The arrows indicate small lines originating from $\mathrm{O}_{2}$.

cause the $\mathrm{FC}$ factor is orders of magnitude smaller than for the $0-0$ transition in the ${ }^{2} \Sigma^{2}{ }^{2} \Pi$ absorption band. Therefore a very strong laser, like the tunable $\mathrm{KrF}$ laser, is required for sufficient excitation. This is clearly seen from the inset in Fig. 2, which shows the linearity of fluorescence intensity with laser power. In this case the $P_{1}(8)$ line is used for excitation. The laser power is varied by inserting several calibrated filters in the beam path of the $\mathrm{KrF}$ laser. Although the applied laser power is in the range of $\mathrm{GW} / \mathrm{cm}^{2}$, the fluorescence intensity is still linearly dependent on the laser power, i.e., even at such high power the $P_{1}(8)$ transition is not saturated. That the saturation limit is not yet reached can also be directly inferred from the strength of the satellite lines that are almost resolved in Fig. 2. In saturation the satellites should be as strong as the main lines which is obviously not true.

The linewidth in Fig. 2 is approximately the linewidth of the excitation laser, which implies that no direct information is obtained about the predissociation lifetime. The only conclusion is that the predissociation lifetime of the $v^{\prime}=3$ state of $\mathrm{OH}\left({ }^{2} \Sigma\right)$ must be longer than 20 ps. The lifetimes of $\mathrm{OH}\left({ }^{2} \Sigma^{+}, v^{\prime}=5,6\right)$ have been determined to be $<10 \mathrm{ps}^{22}$

Figure 3 shows a dispersion spectrum of $\mathrm{OH}$ with a resolution of $\approx 0.4 \mathrm{~nm}$, recorded in a butane flame. $\mathrm{OH}$ is excited on the $Q_{1}(11)$ line, i.e., the quantum state $2 \Sigma$, $v^{\prime}=3, N=11$ is prepared and the subsequent emission is spectrally dispersed. The wavelength range for different $\mathrm{OH}$ vibrational transitions is approximately indicated in the figure. The dominant emission features are the sets of three single strong lines, appearing around 270, 295, and $328 \mathrm{~nm}$.' The single lines originate directly from the laser prepared state $n=11$ in $v^{\prime}$ $=3$ and are identified in the figure as $P_{1}(12), Q_{1}(11)$, and $R_{1}(10)$. Obviously, the three sets of three single strong lines belong to the vibrational transitions from $v^{\prime}=3$ to $v^{\prime \prime}=3($ at $328 \mathrm{~nm}), v^{\prime \prime}=2($ at $297 \mathrm{~nm})$, and $v^{\prime \prime}=$ 1 (at $270 \mathrm{~nm}$ ). From such data the Einstein $A$ coeffi- 


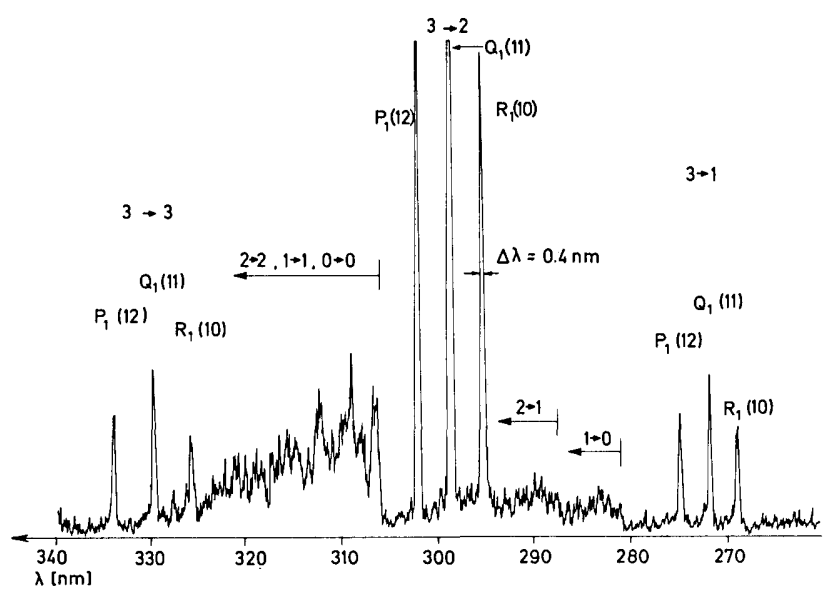

Figure 3. $\mathrm{OH}$ dispersion spectrum for a butane flame. $\mathrm{OH}$ is excited on the $Q_{1}(11)$ line. The spectral resolution is $\approx 0.4 \mathrm{~nm}$. The spectral range of different vibrational bands for the $\mathrm{OH}\left({ }^{2} \sum-2 \Pi\right)$ transition is indicated. The $Q_{1}(11)$ and $P_{1}(12)$ lines are off scale.

cients can be determined, if the sensitivity of the spectrometer setup is eliminated. It should be noted that in the present case the minor effect of fluorescence quenching is not important, because only relative values of Einstein $A$ coefficients are determined.

The $A$ coefficients are measured relative to the 3-2 transition, which we set to 500 , and are compared to various other literature data in Table IV. There is qualitative agreement with some authors, but disagreement with others (compare values for $3-1$ and $3-3)$. To our knowledge the FC factors for $3-4$ and $3-5$ have not been determined before.

The relative emission intensities of $P, Q$, and $R$ lines are not in agreement with the known Hönl-London factors. The $Q$ lines should be considerably stronger than $P$ and $R$ lines. ${ }^{23}$ Because this is true for all vibrational transitions from $\mathrm{OH}\left({ }^{2} \Sigma^{+}, v^{\prime}=3\right)$, the mechanism of fluorescence trapping can be eliminated as an explanation. Instead, the effect is simply explained by the directional properties of light emission and absorption. The present laser is linearly polarized along a horizontal axis, the same axis along which we detect the total amount of fluorescence. This implies that, after $Q$ line excitation, the emission of $P$ and $R$ lines is detected more efficiently than the emission on a $Q$ line. After excitation on $P$ or $R$ lines, on the other hand, the emission on the $Q$ line is detected most efficiently. ${ }^{24,25}$

Although it may be surprising that the LIF of $\mathrm{OH}$ in a flame is polarized, similar observations have been made before. ${ }^{26}$ The strong polarization in the present case is clearly explained by the fact that depolarizing effects, like, for example, collisions or stray magnetic fields, are negligible during the very short predissociation lifetime of the $v^{\prime}=3$ state. The polarization effects are so strong that they have to be taken into account in the determination of rotational temperatures (see Sec. IV.D) No such problems are expected if a depolarizer is used between oscillator and amplifier.
Table IV. Einstein Transition Probabilities from This Work Compared with Selected Results from Other Authors ${ }^{a}$

\begin{tabular}{llccc}
\hline & This work & $\begin{array}{c}\text { Crosley } \\
\text { (calc) }\end{array}$ & $\begin{array}{c}\text { Henneker and } \\
\text { Popkie }\end{array}$ & Learner \\
\hline$A_{30}$ & $9.5 \pm 2$ & 11 & 9 & 12 \\
$A_{31}$ & $130 \pm 15$ & 173 & 159 & 148 \\
$A_{32}$ & 500 & 515 & 488 & 466 \\
$A_{33}$ & $140 \pm 20$ & 113 & 116 & 223 \\
$A_{34}$ & $1.65 \pm 0.40$ & - & - & - \\
$A_{35}$ & $3.2 \pm 0.7$ & - & - & - \\
$A_{36}$ & $\leqq 1.0$ & - & - & - \\
\hline
\end{tabular}

a The other data are contained in a table in Ref. 28 , where the value of $A_{00}$ has been set to 1000 . For good comparison we set our value for $A_{32}$ equal to 500 .

Between the single strong lines some weaker and considerably broader emission appears in the range from 280 to $295 \mathrm{~nm}$ and from 305 to $325 \mathrm{~nm}$. This weaker emission originates from fluorescence of lower vibrational states $\left(v^{\prime}=2,1,0\right)$ of $\mathrm{OH}\left({ }^{2} \Sigma\right)$. The important difference between the direct emission from $v^{\prime}=3$ and lower vibrational states is the emission of single rotational lines from $v^{\prime}=3$ and of many overlapping rotational lines from lower vibrational states. This behavior is due to collisions with other species in the flame. Because the $v^{\prime}=3$ state has a short predissociation lifetime, there is only a small redistribution of quantum states within the $v^{\prime}=3$ state. In contrast the emission from lower vibrational states is broad and less structured, ${ }^{27}$ indicating population in many rotational states. This might originate from direct vibrational quenching or from rotational energy transfer in these levels. Because the predissociation lifetime is much longer for lower vibrational states, many more collisions are possible and many more rotational states become populated in lower vibrational states. The integrated relative fluorescence intensity originating from collisionally populated vibrationally excited $\mathrm{OH}$ seems rather high. This is partly due to the enhanced detection sensitivity for lower vibrational states. The $\mathrm{FC}$ factor for the $0-0$ transition is about an order of magnitude larger than for the $3-3$ transition, ${ }^{28}$ in addition to the fact that lower vibrational states fluoresce more because they predissociate less. Nevertheless appreciable vibrational energy transfer is observed; as in some other cases as well. ${ }^{27}$ As will be seen later, vibrational energy transfer is much less pronounced in the $\mathrm{O}_{2}-\mathrm{H}_{2}$ flame (see Fig. 14).

A more quantitative idea about rotational energy transfer can be obtained from Fig. 4, where a section of another dispersion spectrum is shown at an even better resolution of $0.15 \mathrm{~nm}$. In this case an $\mathrm{O}_{2}-\mathrm{H}_{2}$ flame is used. The $Q_{2}(11)$ is used for excitation, i.e., the $N^{\prime}=$ $11, v^{\prime}=3$ state is prepared in ${ }^{2} \Sigma^{+}$. Only the small section of the dispersion spectrum in the range from 295 to $305 \mathrm{~nm}$, which belongs to the $3-2$ vibrational transition, is shown. Again, the three strong lines that dominate the spectrum originate directly from the single laser excited state and are identified as $R_{2}(10)$, $Q_{2}(11)$, and $P_{2}(12)$. In addition, there is a whole series 


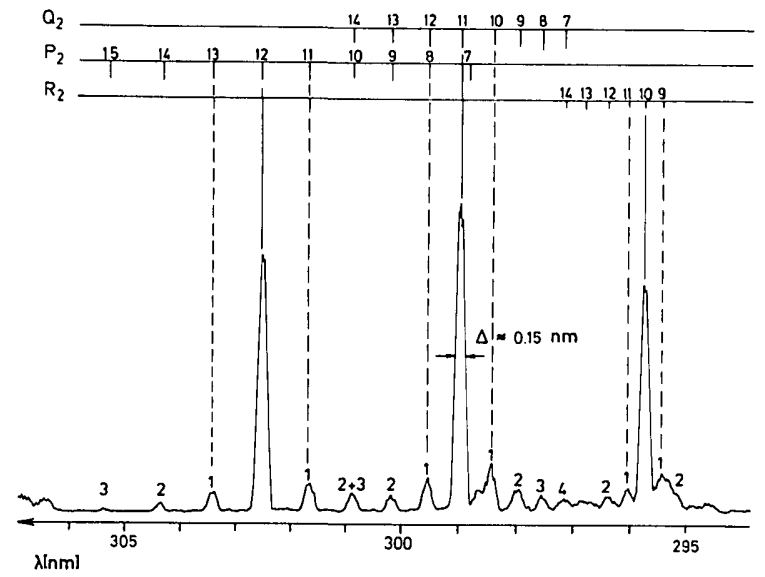

Figure 4. Section of an $\mathrm{OH}$ dispersion spectrum in an $\mathrm{O}_{2}-\mathrm{H}_{2}$ flame at higher resolution. $\mathrm{OH}$ is excited on the $Q_{2}(11)$ line. The resolution is $\approx 0.15 \mathrm{~nm}$. The position of the lines originating directly from the laser prepared state is indicated in the figure by a solid line, the lines originating from the neighboring states with $\Delta N=1$ are indicated by dashed lines. The numbers $1,2,3$ correspond to the value of $\Delta N$. The influence of the directional properties of the fluorescence on the relative intensities of the $P, Q$, and $R$ lines is clearly seen (see text).

of much less intense single rotational lines on both sides of the central peaks, which are also identified in the figure. They originate from the neighboring levels of the $N^{\prime}=11$ state and are populated by rotational energy transfer within $v^{\prime}=3$ in the ${ }^{2} \Sigma^{+}$state. The numbers that are given above the small lines represent the value of $\Delta N$, i.e., the number of rotational quanta transferred from $N^{\prime}=11$. Rotational energy transfer is observed up to $\Delta N=3$. The $P_{2}$ lines at the left-hand side show clearly that the fluorescence intensity decreases with increasing $\Delta N$, a behavior that is expected from a power or energy gap law. ${ }^{29}$ Obviously the $F_{2} \rightarrow$ $F_{1}$ transfer in the ${ }^{2} \Sigma^{+}$state is much slower than the $F_{2}$ $\rightarrow F_{2}$ transfer, the latter being the only transfer process observed here.

Rotational energy transfer within electronically excited states from single laser prepared states have been studied in many experiments. However in these experiments much lower pressures are used to achieve single collision conditions and to avoid secondary collisions. At high pressure the fluorescence becomes broad and unstructured, because many rotational states are populated by secondary collisions and contribute to fluorescence (see, for example, Ref. 27). In the present case the experiment is done at atmospheric pressure. Nevertheless, the direct lines are strongly dominating in the dispersion spectrum, which implies that single collision conditions are valid. Secondary collisions would lead to considerably more redistribution of quantum states.

The collision probability can be estimated from the intensity of the small lines. The integrated intensity in the weak lines is $~ 30 \%$ of the intensity in the main line. Obviously $30 \%$ of the molecules in the prepared $N^{\prime}=11$ states suffered a collision with rotational energy transfer, i.e., the probability $Z$ of such collisions

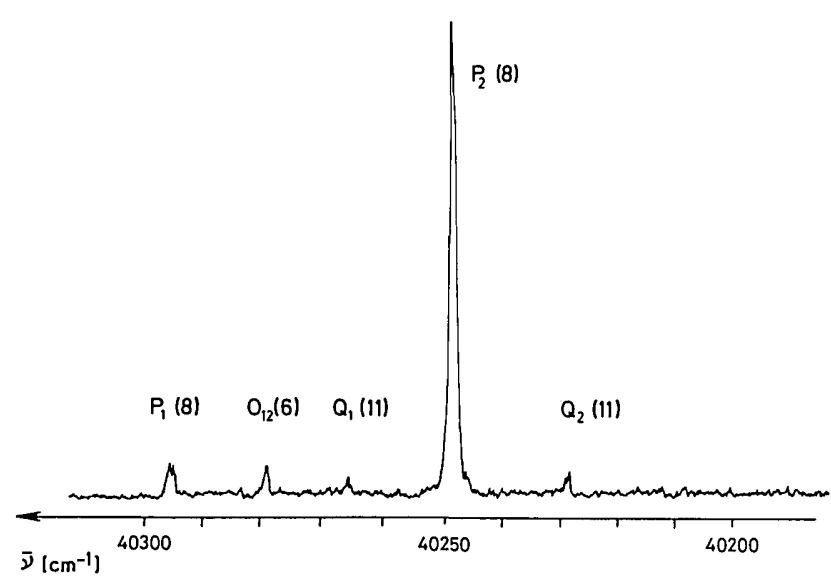

Figure 5. $\mathrm{OH}$ excitation spectrum with the spectrometer set to $297.3 \mathrm{~nm}$ and a rather high resolution $(0.1 \mathrm{~nm})$. Only the $Q_{2}(7) 3-2$ transition is transmitted in emission. This implies that only the fluorescence from the $P_{2}(8), 3-0$ excitation is seen and proves that spectral filtering is possible.

within the predissociation lifetime is 0.3 . On the other hand, the probability of such as collision within $\tau$ is given by

$$
Z=n g \sigma \tau .
$$

If we use the density $n=3 \times 10^{19} / \mathrm{cm}^{3}$ (for normal pressure), a typical relative velocity $g=2 \times 10^{5} \mathrm{~cm} / \mathrm{s}$, a typical cross section for rotational energy transfer of 5 $\times 10^{-16} \mathrm{~cm}^{2}$, this results in a predissociation lifetime of $\approx 100 \mathrm{ps}$. This is a crude estimate, because neither $g$ nor $\sigma$ is well known. In particular the considerably lower density in a flame will tend to increase the estimated lifetime. Nevertheless there is qualitative agreement with theoretical estimates. ${ }^{30}$

An important conclusion is that, although rotational energy transfer takes place even within the short lifetime $\tau_{p}$, most of the emission from $v^{\prime}=3$ is direct. Rotational energy transfer is small even at atmospheric pressures. In contrast to rotational energy transfer, which is directly seen in Fig. 4, fluorescence quenching has not been observed directly. In most cases rotational and vibrational energy transfer as well as fluorescence quenching are of the same order of magnitude. $^{6}$ This suggests a maximum error at 1 bar of $\approx 30 \%$. The actual error will be lower, because only the difference in quenching between $f_{1}$ and $f_{2}$ introduces errors.

Figure 5 shows an $\mathrm{OH}$ excitation spectrum with the spectrometer set to $297.2 \mathrm{~nm}$ with the best available resolution for a butane flame. Obviously the excitation spectrum is very different from Fig. 2: essentially one line is left in the excitation spectrum. This is simply explained by the high resolution of the spectrometer, which is adjusted to transmit only the fluorescence of the $Q_{2}(7)$ line of the $v^{\prime}=3$ to $v^{\prime \prime}=2$ transition. Although different $\mathrm{OH}$ transitions are excited in the scan of the excitation laser, only this single line is seen in emission, demonstrating that the resolution is good enough to separate the fluorescence from 


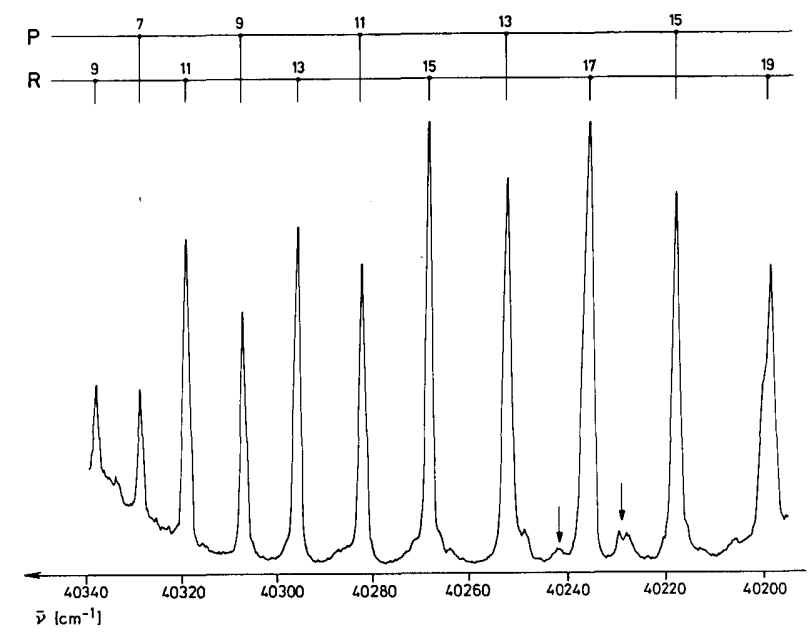

Figure 6. $\mathrm{O}_{2}$ excitation spectrum for the $0-6$ transition in an $\mathrm{O}_{2}-$ $\mathrm{H}_{2}$ flame. The spectrometer is set to $353 \mathrm{~nm}$ with a resolution of $\approx 2$ $\mathrm{nm}$. The arrows indicate weak $2-7$ absorption lines.

single rotational lines. This is of importance for instantaneous temperature field measurements: it is possible to excite two rotational lines simultaneously and to separate the fluorescence from the two excited states by spectral filtering.

\section{B. $\mathrm{O}_{2}$ Spectra}

The various transitions of the $\mathrm{O}_{2}\left(B^{3} \Sigma_{u}^{-}-X^{3} \Sigma_{g}^{-}\right)$Schumann-Runge band that can be excited with the $\mathrm{KrF}$ laser are given in Table II. In this paper we present data only about the most intense vibrational transitions $0-6,2-7$, and $1-6$.

In Fig. 6 the spectrometer is set to $353 \mathrm{~nm}$ with a resolution of $\approx 2 \mathrm{~nm}$. In this wavelength range essentially the vibrational transition $0-15$ of $\mathrm{O}_{2}$ is monitored, although there may be some contributions of the 2-16 transition (see Table III). The excitation spectrum is recorded in an $\mathrm{O}_{2}-\mathrm{H}_{2}$ flame $\approx 10 \mathrm{~cm}$ above the burner tip. The excitation spectrum shows clear rotational structure, which can be analyzed using known spectroscopic data. ${ }^{9}$ The transitions belong to the excitation of $\mathrm{O}_{2}$ from $v^{\prime \prime}=6$ to $v^{\prime}=0$, i.e., highly vibrationally excited $\mathrm{O}_{2}$ is detected in the flame. Even higher vibrationally excited $\mathrm{O}_{2}$ has been observed in other studies. ${ }^{31}$ Only relatively low rotational states can be probed with the $\mathrm{KrF}$ laser $[R(9)-R(19)$ and $P(7)-P(15)]$. Because of the presence of two identical nuclei having no nuclear spin only odd transitions are possible in $\mathrm{O}_{2}$. Some smaller lines in this spectrum (indicated by arrows) belong to the $v^{\prime \prime}=7$ to $v^{\prime}=2$ excitation. These lines appear weakly, because at a resolution of $\approx 2 \mathrm{~nm}$ some fluorescence from $v^{\prime}=2$ to $v^{\prime \prime}$ $=16$ is transmitted by the spectrometer. The linewidth of $\approx 2 \mathrm{~cm}^{-1}$ is obviously larger than the linewidth of the laser. However, the increased linewidth is not due to predissociation; the predissociation lifetimes of $\mathrm{O}_{2}$ are known and only give rise to a linewidth of $0.1 \mathrm{~cm}^{-1} .9$ The large width is simply due to the unresolved spin splittings in the two triplet electronic states.

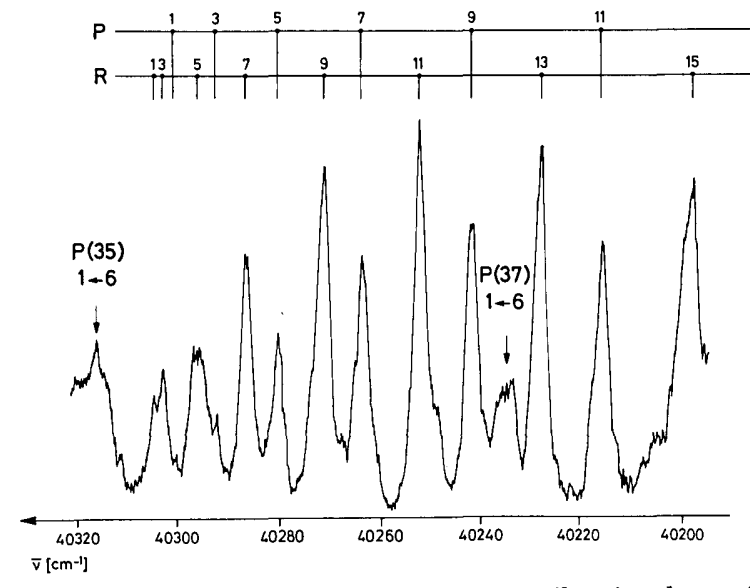

Figure 7. $\mathrm{O}_{2}$ excitation spectra for the $2-7$ vibrational transition in an $\mathrm{O}_{2}-\mathrm{H}_{2}$ flame. The spectrometer is set to $439 \mathrm{~nm}$ with a resolution of $\approx 2 \mathrm{~nm}$. Also two lines of the $1-6$ band are seen.

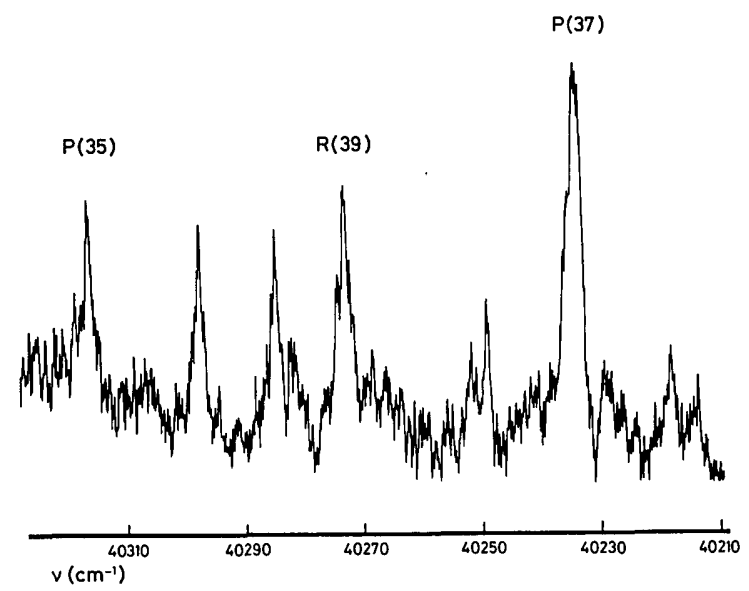

Figure 8. $\mathrm{O}_{2}$ excitation spectra for the $1-6$ vibrational transition in an $\mathrm{O}_{2}-\mathrm{H}_{2}$ flame. The spectrometer is set to $382.3 \mathrm{~nm}$ with a resolution of $\approx 0.5 \mathrm{~nm}$.

Figure 7 shows an $\mathrm{O}_{2}$ excitation spectrum, taken in the same conditions, but with the spectrometer set to $439 \mathrm{~nm}$ and a resolution of $\approx 2 \mathrm{~nm}$. With these settings the spectrometer transmits the $\mathrm{O}_{2}$ fluorescence from $v^{\prime}$ $=2$ to $v^{\prime \prime}=21$ (compare Table III). Again, clearly resolved rotational structure is found in the excitation spectrum. The lines are identified in the figure. The noise is beginning to show up in the spectrum.

The directly observed fluorescence intensity for this $2-7$ excitation spectrum is $\approx 10$ times weaker than for the preceding $0-6$ excitation spectrum. If the different predissociation lifetimes in $v^{\prime}=0$ and $v^{\prime}=2$ (Ref.9) and the $\mathrm{FC}$ factors for the $0-15$ and $2-21$ vibrational transitions are taken into account, ${ }^{20}$ this implies, that $\sim 2$ times more $\mathrm{O}_{2}$ is in $v^{\prime \prime}=6$ than in $v^{\prime \prime}=7$, in approximate agreement with other experiments. ${ }^{31}$

For completeness, we show in Fig. 8 an $\mathrm{O}_{2}$ excitation spectrum for the $1-6$ vibrational transition. Now the spectrometer is set to $328.3 \mathrm{~nm}$ with a fairly high resolution of $0.5 \mathrm{~nm}$ to detect only the $\mathrm{O}_{2} 1-17$ emission. The fluorescence intensity from $v^{\prime}=1$ is rela- 


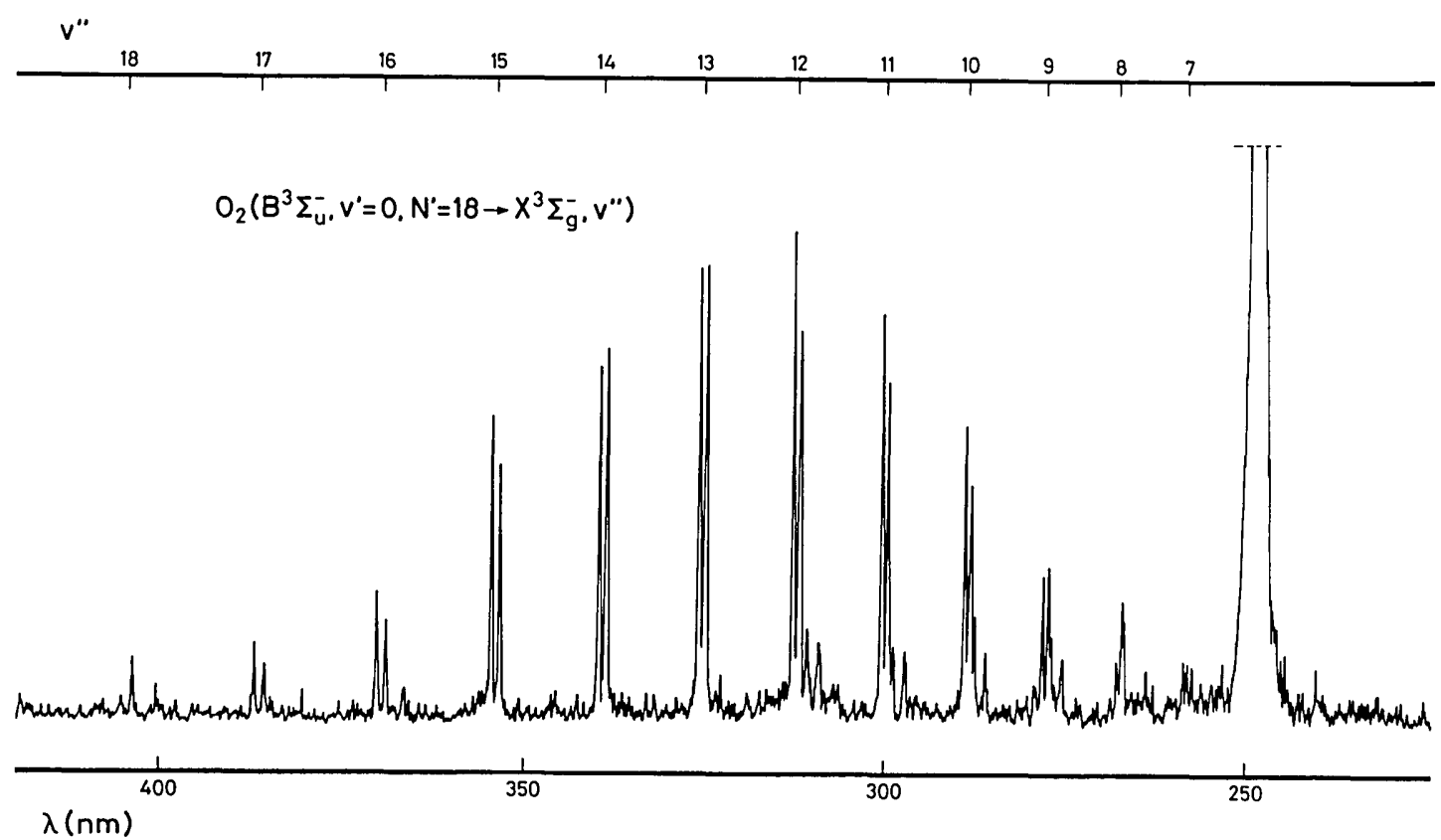

Figure 9. $\mathrm{O}_{2}$ dispersion spectrum for an $\mathrm{O}_{2}-\mathrm{H}_{2}$ flame. $\mathrm{O}_{2}$ is excited on the $R(17)$ line. The spectral resolution is $\approx 0.4 \mathrm{~nm}$. The different peaks correspond to the vibrational transitions originating in the $v^{\prime}=0$ state in $\mathrm{O}_{2}\left(B^{3} \sum_{u}^{-}\right)$, ending in various $v^{\prime \prime}$ states of the ground state. $P$ and $R$ lines appear as doublets in emission.

tively weak, because the $v^{\prime}=1$ state is much more strongly predissociated than the $v^{\prime}=0$ and $v^{\prime}=2$ states. $^{9}$ This spectrum again contains contributions from other absorption lines that are simultaneously excited. Nevertheless the main three lines in the spectrum are clearly identified as $P(35), P(37)$, and $R(39)$. The high rotational lines of this vibrational transition allow us to measure rotational temperatures of $\mathrm{O}_{2}$ with one laser by spectral filtering of fluorescence. For example, simultaneous excitation of the $R(17)$ line in the $0-6$ and the $P(37)$ line in the $1-6$ vibrational band can be achieved with a somewhat broader laser (these lines are only $0.8 \mathrm{~cm}^{-1}$ apart). The fluorescence can be observed separately around $380.5 \mathrm{~nm}$ for $P(37)$, $1-6$ and around $324.5 \mathrm{~nm}$ for $R(17) 0-6$ excitation.

Figure 9 shows a dispersion spectrum of $\mathrm{O}_{2}$ with a resolution of $0.4 \mathrm{~nm}$ for an $\mathrm{O}_{2}-\mathrm{H}_{2}$ flame. The laser is tuned to excite the $R(17), 0-6$ line, i.e., the state $N^{\prime}=$ 18 is prepared in ${ }^{3} \Sigma_{u}^{-}$. The fluorescence obviously extends over a very wide range. The spectrum is shown only in the range from 250 to $400 \mathrm{~nm}$. The big peak at the right is from scattered light of the $\mathrm{KrF}$ laser. A very regular series of different fluorescence lines is clearly seen. This fluorescence originates from a series of vibrational transitions starting in the excited ${ }^{3} \Sigma_{u}^{-}, v^{\prime}=0$ state and ending in the vibrational states $v^{\prime \prime}=7-18$ of the electronic ground state. The relative strength of the lines is due to the different FC factors for the vibrational transitions $0-v^{\prime \prime}$. There is good agreement with the published $\mathrm{FC}$ factors. ${ }^{20}$

The spectrum shows clear rotational structure. It should be emphasized that a spectral resolution of $\approx 0.4 \mathrm{~nm}$ is only sufficient to separate $P$ and $R$ branches in emission, but not to resolve single rotational lines. If all rotational states were populated in the electroni- cally excited $\mathrm{O}_{2}\left({ }^{3} \Sigma_{u}^{-}, v^{\prime}=0\right)$, just a smooth rotational envelope would be expected. The single rotational lines prove that emission occurs only from the single laser excited rotational state. As in the case of $\mathrm{OH}$, the predissociation lifetime is so short that redistribution within the excited state is negligible. The appearance of single rotational lines proves that rotational energy transfer-and therefore also fluorescence quenching-is negligible at atmospheric pressures.

Figure 10 shows a dispersion spectrum of $\mathrm{O}_{2}$ in an $\mathrm{O}_{2}-\mathrm{H}_{2}$ flame after excitation on the $P(9), 2-7$ transition. Because the resolution of the spectrometer is now only $\approx 1 \mathrm{~nm}, P$ and $R$ lines can no longer be separated in emission. The somewhat increased background at $310 \mathrm{~nm}$ is due to the direct continuous $\mathrm{OH}$ emission from the flame; it is seen here because the boxcar gate was not adjusted short enough. It is interesting to note that, in contrast to the smooth behavior of the emission from $v^{\prime}=0$ with one maximum around $320 \mathrm{~nm}$, the emission from $v^{\prime}=2$ has a clear structure with two minima, one around $300 \mathrm{~nm}$ and one around $375 \mathrm{~nm}$. This is due to the nodal structure in the vibrational wave function for $v^{\prime}=2$.

This effect is also seen in the last dispersion spectrum of Fig. 11, which originates from the excitation of the $P(37)$ line of the $1-6$ vibrational transition. The minimum around $340 \mathrm{~nm}$ is due to the node in the $v^{\prime}=$ 1 wave function. The other lines in the figure originating from $v^{\prime}=0$ are due to simultaneous excitation of the $R(17)$ line of the $0-6$ transition.

\section{C. $\mathrm{H}_{2} \mathrm{O}$ Spectra}

Some excitation spectra for $\mathrm{H}_{2} \mathrm{O}\left(C^{1} B_{1}-X^{1} A_{1}\right)$ were recorded in the flame. The excitation of the $C$ state is done with two photons from the tunable $\mathrm{KrF}$ laser. 


$$
O_{2}\left(B^{3} \Sigma_{u}^{-} \cdot v^{\prime}=2 \cdot N^{\prime}=8 \rightarrow X^{3} \Sigma_{g}^{-} \cdot v^{\prime \prime}\right)
$$

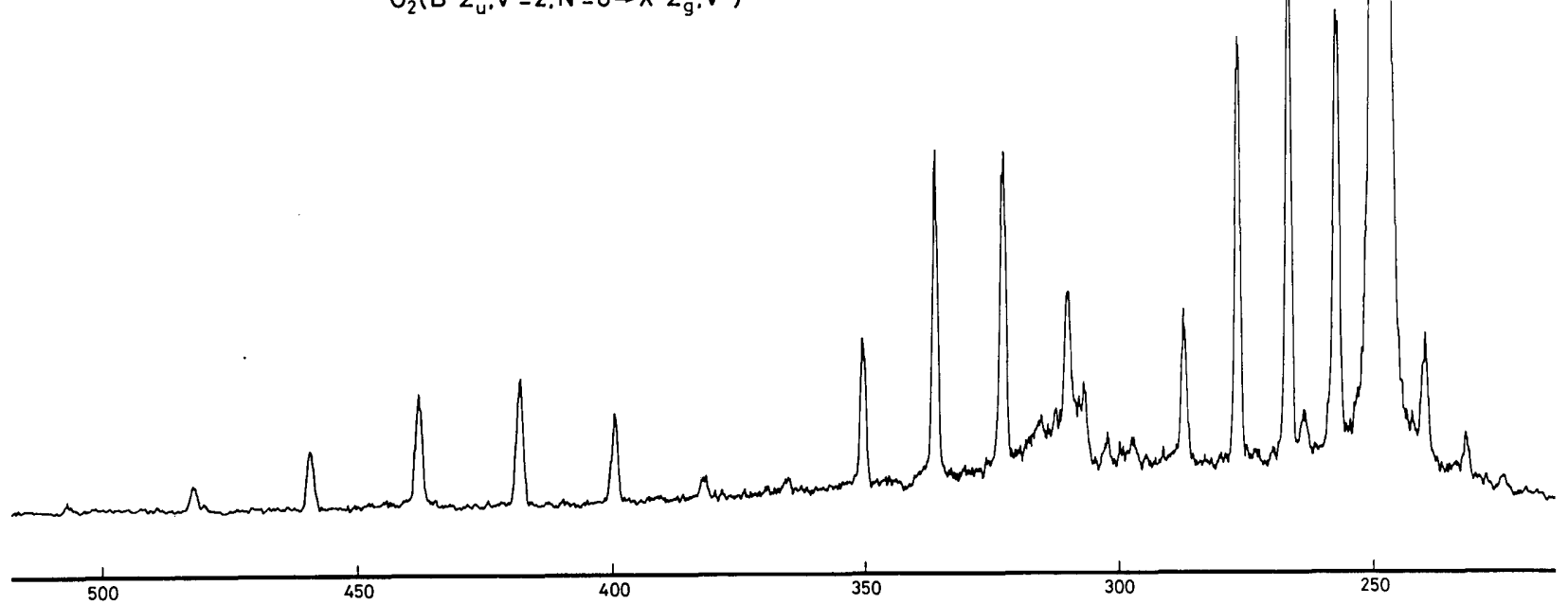

$\lambda(\mathrm{nm})$

Figure 10. $\mathrm{O}_{2}$ dispersion spectrum in an $\mathrm{O}_{2}-\mathrm{H}_{2}$ flame after excitation on the $P(9), 2-7$ line. The nodal structure in the vibrational wave function of the $v^{\prime}=2$ state is directly seen in the intensity distribution.

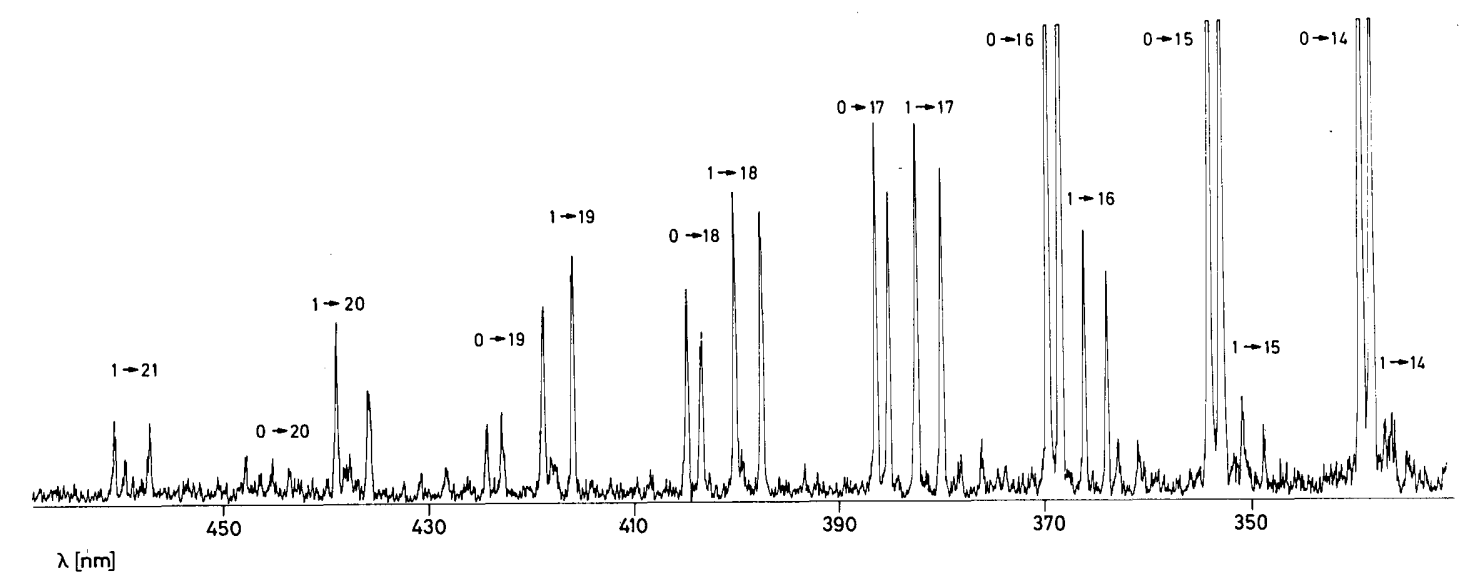

Figure 11. $\mathrm{O}_{2}$ dispersion spectrum for an $\mathrm{O}_{2}-\mathrm{H}_{2}$ flame. $\mathrm{O}_{2}$ is simultaneously excited on the $P(37), 1-6$ and the $R(17), 0-6$ lines.

The $C$ state predissociates via a crossing with the $B$ state and the $B$ state in turn yields electronically excited $\mathrm{OH}$ products in the ${ }^{2} \Sigma^{+}$state. This yields an intense (but long-lived) fluorescence around $308 \mathrm{~nm}$. Despite the fast predissociation to the $B$ state, there is another competing fluorescence from the $C$ state directly to the $A$ state. This bound-free emission appears between 400 and $500 \mathrm{~nm}$ and is only emitted during a short predissociation lifetime of several picoseconds.

Because of the widespread bound-free fluorescence, the spectrometer can be set to any wavelength between 400 and $500 \mathrm{~nm}$. This is very convenient, because it allows us to discriminate against the fluorescence of $\mathrm{O}_{2}$ and $\mathrm{OH}$. The dispersion spectra have been measured before and are not expected to be any different from those in the earlier publication, because the emission is not dependent on the excited rotational state. For details the reader is referred to Refs. 13 and 14.

\section{Temperature Measurements}

In this section we first discuss the measurement of the absolute rotational temperature of $\mathrm{OH}$ in a butane flame, recorded $\approx 2 \mathrm{~cm}$ above the burner tip. As mentioned above, the setting of the central wavelength and the resolution of the spectrometer is very important for these measurements. First the laser is tuned to a particular absorption line and the spectrometer is optimized, with a rather good resolution of $\approx 0.2 \mathrm{~nm}$, to transmit only one of the three strong lines of the $3-2$ 
emission band. To minimize polarization effects, the fluorescence intensity is measured on $P$ or $R$ lines if a $Q$ line is used for excitation and on a $Q$ line if a $P$ or $R$ lines is used for excitation. With both combinations the same directional properties are obtained in the socalled "high $J$ limit," 25 which is a good approximation for the present case.

The measured intensities are corrected for the line strength for absorption and emission ${ }^{23}$ and also for the degeneracy of the ground state levels. These corrected intensities are plotted logarithmically in Fig. 12 against the energy of the ground state levels. The straight line in the plot indicates a well-defined rotational temperature of $\approx 1500 \mathrm{~K}$, a temperature that is in approximate agreement with expectations. The straight line in the plot suggests that predissociation in $v^{\prime}=3$ is not strongly quantum state dependent, because otherwise strong deviations from a straight line are expected. Nevertheless, precise temperature measurements will require a separate calibration procedure, for example, by delayed detection of $\mathrm{OH}$ after 193-nm photolysis of $\mathrm{H}_{2} \mathrm{O}_{2}$ in a high pressure cell.

An easier procedure is used to determine small temperature differences in the flame. In this case only two lines are used to determine the temperature, the $P_{2}(8)$ and the $R_{1}(15)$ lines of the 3-0 vibrational transition. These lines are very close in their excitation wavelength $\left(\approx 3.1 \mathrm{~cm}^{-1}\right)$ and are for several reasons ideally suited for instantaneous temperature field measurements. First, simultaneous excitation of these lines is possible with an easily achieved increased laser linewidth. Second, the probed energy levels are almost $3000 \mathrm{~cm}^{-1}$ apart in energy, which implies that large population differences are expected for rather small changes in temperature. In addition the fluorescence from these levels can be nicely separated by narrow bandwidth filters. With the very strong $\mathrm{KrF}$ laser, a large sheet in the flame can be illuminated with sufficient intensity and the resulting fluorescence can be viewed from the side with two image intensifiers, one equipped with a filter to record the fluorescence from $P_{2}(8)$ excitation and the other one equipped with another filter to record the fluorescence from $R_{1}(15)$ excitation. From the relative fluorescence intensities it should be possible to determine temperature fields on the basis of a single laser shot.

Because there were no image intensifiers available, we used the following procedure. The spectrometer is fixed to $300.2 \mathrm{~nm}$ with a resolution of $\approx 1 \mathrm{~nm}$. This wavelength range is chosen to obtain optimum transmission for the $Q_{1}(16), 3-2$ emission, which follows $\mathrm{OH}$ excitation on the $R_{1}(15)$ line. A small fraction of the fluorescence following $P_{2}(8)$ excitation [the $P_{2}(8)$, $3-2$ emission] is also transmitted in this wavelength range.

Now the laser is scanned only over the narrow range containing the $R_{1}(15)$ and the $P_{2}(8)$ lines. Such laser scans are made at different positions in the flame and three of them are shown in Fig. 13. Because the probed energy levels $\left[P_{2}(8):{ }^{2} \Pi_{1 / 2}, N^{\prime \prime}=8, R_{1}(15)\right.$ : $\left.{ }^{2} \Pi_{3 / 2}, N^{\prime \prime}=15\right]$ are almost $3000 \mathrm{~cm}^{-1}$ apart, the ratio of

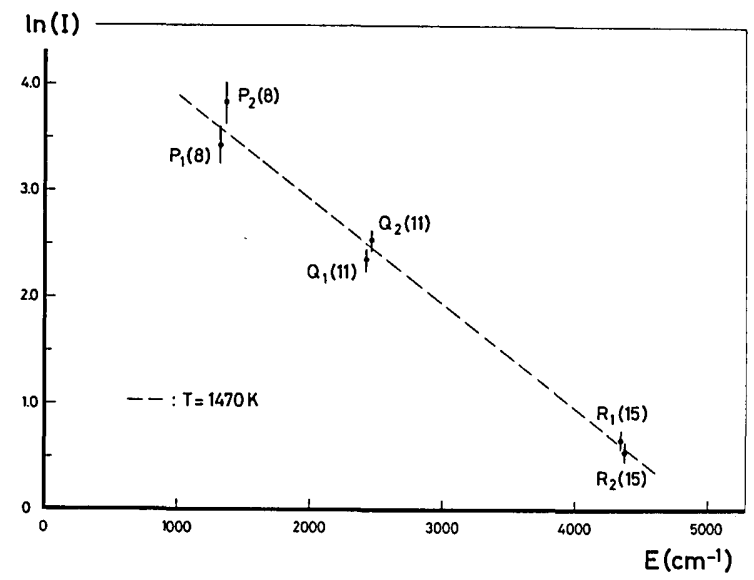

Figure 12. Absolute temperature of $\mathrm{OH}$ in a butane flame, determined from the logarithmic plot of the emission intensity against the energy of the ground state levels. The different $\mathrm{OH}$ transitions used are indicated in the figure.

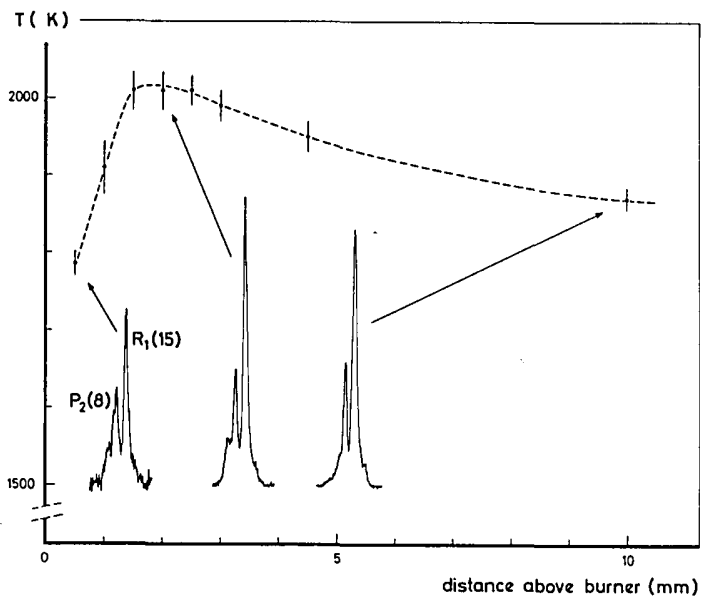

Figure 13. Variation of the rotational temperature of $\mathrm{OH}$ with the distance above the burner tip in a butane flame. Temperature differences are measured with an accuracy of $\approx 1 \%$ of the absolute temperature by comparing the emission intensity following $P_{2}(8)$ and $R_{1}(15)$ excitation.

the corresponding fluorescence intensities is very sensitive to small changes in temperature. Even temperature differences of $20 \mathrm{~K}$, corresponding to only $\approx 1 \%$ of the absolute temperature, can be measured. To determine absolute temperatures, the attenuation of the $P_{2}(8)$ line at the special settings of the spectrometer is measured and included in the analysis.

A similar procedure is followed to determine the rotational temperature of $\mathrm{O}_{2}\left(v^{\prime \prime}=6\right)$. Now, the $R(39)$, $1-6$ and the $R(15), 0-6$ absorption lines, which are $<5 \mathrm{~cm}^{-1}$ apart, are used for excitation. Accurate rotational temperatures could be determined, even far above the burner tip with considerably colder $\mathrm{O}_{2}\left(v^{\prime \prime}=\right.$ $6)$.

It should be noted that the noise in the temperature data is essentially due to pulse-to-pulse fluctuations in laser power and to instabilities in the flame. If temperatures are, however, determined in a single laser 

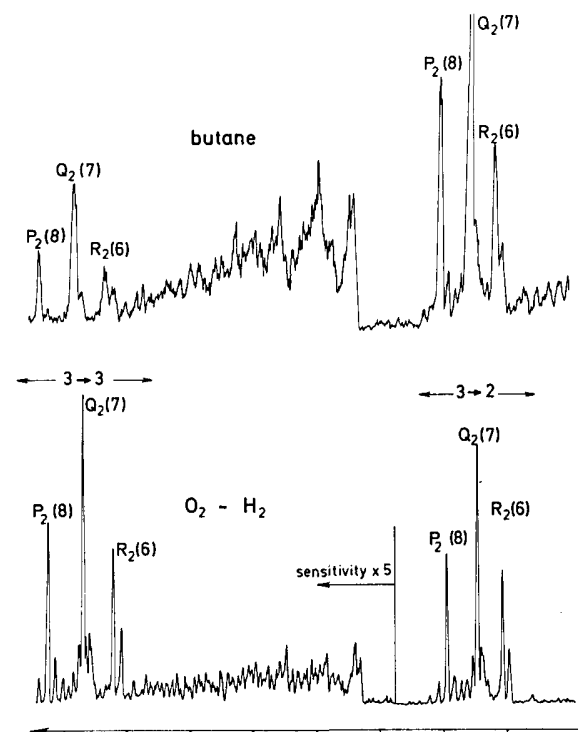

$\begin{array}{llll}330 & 320 & 310 & 300 \\ \lambda(\mathrm{nm}) & & & \end{array}$

Figure 14. Comparison of $\mathrm{OH}$ dispersion spectra for an $\mathrm{O}_{2}-\mathrm{H}_{2}$ flame and a butane flame excited on the $P_{2}(8)$ line and recorded with a resolution of $0.2 \mathrm{~nm}$. In the lower trace, the sensitivity has been changed by a factor of 5 at $\approx 304 \mathrm{~nm}$.

shot, these sources of error are completely eliminated. The relative fluorescence yield is only determined by the rotational temperature in the flame. In contrast to density measurements, which require the analysis of absolute intensities, temperature measurements require only relative intensities and are therefore much easier and much more precise.

Finally, we want to demonstrate that information about vibrational energy transfer in $\mathrm{OH}\left({ }^{2} \Sigma^{+}\right)$can be obtained from such data. Fig. 14 shows a comparison of two dispersion spectra of $\mathrm{OH}$, both excited on the same $P_{2}(8)$ line. The upper trace shows the dispersion spectrum for a butane flame, the lower trace the spectrum for an $\mathrm{O}_{2}-\mathrm{H}_{2}$ flame. The dispersion spectra are clearly different in the 308-325-nm range, where emission from lower vibrational states occurs. This is best seen in comparison with the $R_{2}(6)$ line from the $3-3$ vibrational transition at $326 \mathrm{~nm}$. For the $\mathrm{O}_{2}-\mathrm{H}_{2}$ flame the fluorescence intensity is $\approx 5$ times weaker than the $R_{2}(6)$ line, whereas for the butane flame the maximum emission from lower vibrational states is twice as large. Obviously much more vibrational energy transfer takes place in a butane flame.

\section{v. Discussion and Conclusions}

It is demonstrated that tunable high power excimer lasers can be used to excite efficiently various transitions in $\mathrm{OH}, \mathrm{O}_{2}$, and $\mathrm{H}_{2} \mathrm{O}$. Most of the spectroscopic information about these transitions is well known and has been used to identify unambiguously the excitation and dispersion spectra. For the first time the 30 vibrational transition in the ${ }^{2} \Sigma^{+}-^{2} \Pi$ absorption band of $\mathrm{OH}$ has been excited. Vibrationally hot $\mathrm{O}_{2}$ has been detected via the $\mathrm{O}_{2}$ Schumann-Runge absorption bands. It is successfully demonstrated that, despite the many different transitions in the tuning range of the $\mathrm{KrF}$ laser and despite the complicated gas composition in the flame, the excitation spectra of $\mathrm{OH}, \mathrm{O}_{2}$, and $\mathrm{H}_{2} \mathrm{O}$ can be measured separately. From dispersion spectra information can be obtained about vibrational and rotational energy transfer and about FC factors.

For all transitions a short predissociation lifetime in the upper electronic state is found. This allows LIF at higher pressures without the typical problems of fluorescence quenching. Although the short predissociation lifetimes reduce the fluorescence yield dramatically, the emitted fluorescence intensity is still very high, partly due to the larger densities at higher pressures and partly due to the efficient excitation with the very strong tunable $\mathrm{KrF}$ laser. The experiment shows that sufficient LIF signal is obtained from very small fluorescence volumes, even when a large volume is illuminated. Therefore instantaneous temperature field measurements should be possible with this method. The present experimental setup is however not adequate for quantitative temperature measurements. Experiments with planar LIF (Ref. 5) and an image intensifier are in progress.

For both $\mathrm{OH}$ and $\mathrm{O}_{2}$ it should be possible to do instantaneous temperature measurements with only one laser because the fluorescence from simultaneously excited levels can be separated by spectral filtering. Because only one tunable excimer laser with somewhat increased bandwidth is required in this case this implies moderate effort compared to other methods. For example, for the simultaneous excitation of $\mathrm{OH}\left({ }^{2} \Sigma^{+}, v^{\prime}\right.$ $\left.=3 \leftarrow 2 \Pi, v^{\prime \prime}=0\right)$ via the $P_{2}(8)$ and $R_{1}(15)$ lines, the emission lines are sufficiently spread in wavelength to be separated by narrowband filters. For $\mathrm{O}_{2}$, the $P(37)$ line of the $1-6$ transition and the $R(17)$ line of the $0-$ 6 transition can be excited simultaneously and the corresponding fluorescence can again be separated by filters. This allows the measurement of rotational temperatures for $\mathrm{O}_{2}$ and $\mathrm{OH}$ with one laser on a single shot basis.

In summary, the method of LIF with a high power tunable excimer laser via fast predissociating states is a promising tool to make instantaneous temperature field measurements.

\section{References}

1. U. Hefter and K. Bergmann, "Spectroscopic Detection Methods," in Atomic and Molecular Beam Methods, G. Scoles, Ed. (publisher, New York, 1986).

2. M. N. R. Ashfold, "Multiphoton Probing of Rydberg states," Mol. Phys. 58, 1 (1986); D. H. Parker, "Laser Ionisation Spectroscopy and Mass Spectrometry," in Ultrasensitive Laser Spectroscopy (Academic, New York, 1983).

3. S. A. J. Druet and J. P. E. Taran, "CARS Spectroscopy," Prog. Quantum Electron. 7, 1 (1981).

4. M. B. Long, P. S. Levin, and D. C. Forguette, "Simultaneous Two-Dimensional Mapping of Species Concentration and Temperature in Turbulent Flames," Opt. Lett. 10, 267 (1985); R. W. Dibble and R. E. Hollenbach, "Laser Rayleigh Thermometry in Turbulent Flames," in Proceedings, Eighteenth Symposium (International) on Combustion (The Combustion Institute, 
Pittsburgh, PA, 1981), p. 1489.

5. J. M. Seitzman, G. Kychakoff, and R. K. Hanson, "Instantaneous Temperature Field Measurements Using Planar LaserInduced Fluorescence," Opt. Lett. 10, 439 (1985).

6. R. A. Copeland, M. J. Dyer, and D. R. Crosley, "Rotational Level Dependent Quenching of $\mathrm{A}^{2} \Sigma^{+} \mathrm{OH}$ and OD,"J. Chem. Phys. 82, 4022 (1985).

7. G. Herzberg, Molecular Spectra and Molecular Structure, Vols. 1 and 2 (Van Nostrand, New York, 1950).

8. P. B. Kelly and B. S. Hudson, "Resonance Fluorescence and Resonance Raman Spectra of Molecular Oxygen," Chem. Phys. Lett. 114, 451 (1985).

9. M. P. Lee and R. K. Hanson, "Calculations of $\mathrm{O}_{2}$ Absorption and Fluorescence at Elevated Temperatures for a Broadband Argon-Fluoride Laser Source at 193 nm," J. Quant. Spectrosc. Radiat. Transfer 36, 425 (1986).

10. G. H. Diecke and H. M. Crosswhite, "The UV Bands of OH: Fundamental Data," J. Quant. Spectrosc. Radiat. Transfer 2, 97 (1962).

11. D. M. Creek and R. W. Nicholls, "A Comprehensive Reanalysis of the $\mathrm{O}_{2}$ Schumann-Runge Band System," Proc. R. Soc. London Ser. A 341, 517 (1975).

12. M. P. Docker, A. Hodgson, and J. P. Simons, "Molecular Emission from $\mathrm{H}_{2} \mathrm{O} / \mathrm{D}_{2} \mathrm{O}$ and Photodissociation Dynamics on the $\mathrm{B}-\mathrm{A}$ Surface," Mol. Phys. 57, 129 (1986).

13. G. Meijer, J. J. ter Meulen, P. Andresen, and A. Bath, "Sensitive Quantum State Selective Detection of $\mathrm{H}_{2} \mathrm{O}$ and $\mathrm{D}_{2} \mathrm{O}$ by $(2+1)$ Resonance Enhanced Multiphoton Ionisation," J. Chem. Phys. 85, 6914 (1986).

14. V. Engel, G. Meijer, A. Bath, P. Andresen, and R. Schinke, "The C-A Emission in Water: Theory and Experiment," J. Chem. Phys. 87, 3410, (1987).

15. J. E. Frederick and R. D. Hudson, "Predissociation Linewidth and Oscillator Strength for the $(2,0)$ to $(13,0)$ Schumann-Runge Bands of $\mathrm{O}_{2}$," J. Mol. Spectrosc. 74, 247 (1979).

16. R. J. Cattolica, "OH Rotational Temperature from Two-Line Laser-Excited Fluorescence" Appl. Opt. 20, 1156 (1981).

17. R. P. Lucht, N. M. Laurendeau, and D. W. Sweeney, "Temperature Measurement by Two-Line Laser-Saturated OH Fluorescence in Flames," Appl. Opt. 21, 3729 (1982).
18. D. Weide, P. Andresen, and H. J. Freund, "UV Laser Stimulated Resonant Desorption from Metal Surfaces: NO/Ni (100)," Chem. Phys. Lett. 136, 106 (1987).

19. K. Shibuya and F. Stuhl, "Single Vibronic Emissions from NO $\mathrm{B}^{2} \Pi,\left(\mathrm{v}^{\prime}=7\right)$ and $\mathrm{O}_{2} \mathrm{~B}^{2} \sum_{u}^{-}\left(\mathrm{v}^{\prime}=4\right)$ excited by 193-nm ArF Laser," J. Chem. Phys. 76, 1184 (1982).

20. R. Harris, M. Blackledge, and J. Generosa, "Rydberg-KleinRees (RKR) Franck-Condon Factors for the O SchumannRunge System Including High Vibrational Quantum Numbers," J. Mol. Spectrosc. 30, 506 (1969).

21. I. Kovacs, Rotational Structure in the Spectra of Diatomic Molecules (Adam Hilger, London, 1969).

22. C. Carlone and F. W. Dalby, "Spectrum of the Hydroxyl Radical," Can. J. Phys. 47, 1945 (1969).

23. I. L. Chidsey and D. R. Crosley, "Calculated Rotational Transition Probabilities for the A-X System of OH," J. Quant. Spectrosc. Radiat. Transfer 23, 187 (1980).

24. C. H. Greene and R. N. Zare, "Determination of Product Populations and Alignment Using Laser-Induced Fluorescence," J. Chem. Phys. 78, 6741 (1983).

25. R. Altkorn and R. N. Zare, "Effects of Saturation on LaserInduced Fluorescence Measurements of Population and Polarisation," Ann. Rev. Phys. Chem. 35, 265 (1984).

26. P. M. Doherty and D. R. Crosley, "Polarization of Laser-Induced Fluorescence in $\mathrm{OH}$ in an Atmospheric Pressure Flame," Appl. Opt. 23, 713 (1984).

27. R. K. Lengel and D. R Crosley, "Energy Transfer in $\mathrm{A}^{2} \Sigma^{+}$. II Vibrational," J. Chem. Phys. 68, 5309 (1978).

28. D. R. Crosley and R. K. Lengel, "Relative Transition Probabilities and the Electronic Transition Moments in the A-X System of OH," J. Quant. Spectrosc. Radiat. Transfer 15, 579 (1975).

29. H. W. Lulf, "Author, Add Title to the Galleys," Ph.D. Thesis, U. Gottingen (1986).

30. M. L. Sink, A. D. Bandrauk, and R. Lefevre, "Theoretical Analysis of the Predissociation of the A State of OH," J. Chem. Phys. 73, 4451 (1980).

31. R. A. Copeland, P. C. Cosby, D. R. Crosley, J. P. Jeffries, and T. G. Slanger, "Vibrationally Excited $\mathrm{O}_{2}$ in Flames: Measurements on $\mathrm{v}^{\prime \prime}=9-11$ by Laser-Induced Fluorescence," J. Chem. Phys. 86, 2500 (1987).
"I never write
'metropolis' for
seven cents
because I can
get the same
price for 'city'."

Samuel

Clemens 